\title{
Integración de redacciones en los medios locales y comarcales catalanes
}

Recibido: 2/03/2010

\author{
Pere MASIP MASIP \\ Universitat Ramon Llull \\ peremm@blanquerna.url.edu \\ Josep Lluís Micó SANZ \\ Universitat Ramon Llull \\ JosepLluisMS@blanquerna.url.edu \\ Carles Ruiz CABALlERO \\ Universitat Ramon Llull \\ carlesrc@blanquerna.url.edu \\ Sonia GonzÁLEz MOLINA \\ Universitat de Vic \\ Sonia.gonzalez3@uvic.cat \\ David DOMINGo SANTAMARÍA \\ Universitat Rovira I Virgili \\ Anna NoguÉ RegÁs \\ Agència Catalana de Notícies \\ anogue@telefonica.net
}

Aceptado: 26/10/2010

\begin{abstract}
Resumen
Se ofrecen los resultados obtenidos del análisis de la organización de las redacciones de los medios de proximidad catalanes en el marco de la convergencia periodística. Los datos se han obtenido a partir de una encuesta distribuida entre los 123 medios que forman parte de la Associació Catalana de la Premsa Comarcal (ACPC), con un índice de respuesta del 45\%, y de 19 entrevistas en profundidad mantenidas con periodistas y directivos de 8 grupos de comunicación. Las empresas fueron seleccionadas según criterios de distribución geográfica, diversidad mediática, periodicidad y tirada.Se observa cómo la convergencia periodística no supone necesariamente la integración de redacciones, aunque habitualmente sí que se desarrolla alguna forma de producción integrada. Las fórmulas desarrolladas son diversas, con distintos niveles de integración.
\end{abstract}

Palabras clave: Convergencia periodística, Integración de redacciones, Prensa local, Cataluña.

\section{Newsroom integration in Catalan local newspapers}

\begin{abstract}
The aim of this article is examine the characteristics of newsrooms' organization of Catalan local media as a part of media convergence. Data was obtained from a survey distributed among media members of the Catalan Association of Local Newspapers, with a response rate of 45\%, and 19 in-depth interviews with journalists and managers of 8 media groups. Companies were selected taking into account a wide range of variables: geographical distribution, periodicity, circulation, etc. Results show media convergence does not mean newsroom integration, but media groups usually develop some kind of integrated production. Formulas developed by media are diverse, with different levels of integration. Key words: Media convergence, newsroom integration, local media, Catalonia.
\end{abstract}

\section{Referencia normalizada}

MASIP MASIP, Pere et al (2011): "Integración de redacciones en los medios locales y comarcales catalanes”. Estudios sobre el mensaje periodístico. Vol. 17, núm. 1, págs.: 167-182. Madrid, Servicio de Publicaciones de la Universidad Complutense.

Sumario: 1. Introducción. 2. Prensa local: concentración empresarial y multimedia. 3. Objetivos y metodología. 4. Resultados. 4.1. Fábricas de información. 4.2. Producción periodística y organización de las redacciones. 4.2.1. Redacciones unificadas. 4.2.2. Redacciones coordinadas. 4.2.3. Redacciones integradas a la fuerza. 5. Actitudes y estrategias ante la convergencia. 6. Conclusiones. 7. Referencias bibliográficas. 


\section{Introducción}

Las transformaciones en el ámbito del periodismo siempre han sido constantes, pero nunca antes habían alcanzado un nivel de aceleración como el actual. Las innovaciones tecnológicas que se han instaurado en las empresas de comunicación han llegado a tambalear algunos de los paradigmas clásicos de la profesión, han impulsado la implantación de nuevas figuras como el periodista polivalente, han otorgado un rol más activo a las audiencias, han resquebrajado los tradicionales límites entre medios y han activado nuevas formas de concebir las redacciones.

En este contexto, una de las palabras de moda entre profesionales y académicos es convergencia -o cualquiera de las variantes que suele adoptar: confluencia, fusión de redacciones, coordinación, producción cross-media..., y que sugieren distintos grados de desarrollo-.

Como ya se han encargado de recordar diversos autores, la convergencia es un fenómeno complejo que puede adoptar formas y tener características distintas. Sería un error creer que es sólo un simple efecto de la tecnología (DUPAGNE y GARRISON, 2006), sino que está sujeta a condiciones específicas de diversa índole (profesionales, económicas...) (BoczKOWSKI y FERRIs, 2005).

Siguiendo clasificaciones consolidadas como la propuesta por DAILEY et al. (2005), con frecuencia la convergencia es percibida como un proceso que culmina con la integración de redacciones, el supuesto ideal a alcanzar. La realidad sin embargo es muy distinta y, como recuerdan SAlaVerRía y Negredo (2009), la convergencia y la integración de redacciones no son lo mismo. Mientras que la primera se refiere a un proceso de acercamiento -material o funcional- entre espacios tradicionalmente distantes, la segunda comporta el trabajo en un mismo espacio físico y bajo una dirección editorial única de equipos redaccionales que hasta el momento lo hacían de forma independiente.

GARCía AviLÉs et al (2009), a partir del análisis de medios españoles, alemanes y austriacos, identifican tres modelos de convergencia de redacciones: integración plena, colaboración y coordinación. La integración plena exige la existencia de una redacción única que produce para diversas plataformas bajo un mando editorial único y donde la producción y la distribución se subordinan a la actualidad informativa. La colaboración entre redacciones no demanda la existencia de una redacción única, aunque puede existir, sino que el trabajo se estructura en torno a una mesa multimedia que coordina la labor diaria de las redacciones y los flujos informativos. Finalmente, en la coordinación de soportes aislados, las redacciones permanecen separadas y los contactos entre ellas no están institucionalizados, dejándose en manos de los propios periodistas.

En cualquier caso, parece claro que la convergencia incide en mayor o menor grado en la organización de las redacciones. Una reorganización encaminada a mejorar la eficiencia en la producción de contenidos periodísticos, gracias, por ejemplo, a la planificación conjunta de la cobertura de hechos noticiosos y al uso de los soportes más adecuados para cada momento; o la producción centralizada de contenidos para distintos medios y su distribución multiplataforma. 
Como se verá más adelante con detalle, el objetivo de este trabajo es mostrar las principales iniciativas de convergencia desarrolladas en los medios de proximidad catalanes y describir su incidencia en la organización de las redacciones.

\section{Prensa local: concentración empresarial y multimedia}

Resulta imposible identificar un único motivo que explique por qué un número creciente de medios están apostando por la convergencia periodística, en cualquiera de sus formas. Sin embargo, el fenómeno no se habría desencadenado si no se hubieran dado las condiciones pertinentes, articuladas en dos ejes básicos: la digitalización y las nuevas estructuras empresariales.

Desde los años 90 se ha venido produciendo un proceso de concentración empresarial y de diversificación mediática. De la filosofía monomedia, se pasaba a la multimedia. Grupos editores de periódicos desembarcaban en el mundo audiovisual a la búsqueda de nuevas oportunidades de negocio. Prisa, Planeta, Godó, Vocento o Unidad Empresarial son claros ejemplos.

No obstante, la apuesta empresarial hacia la diversificación mediática no es exclusiva de los grupos de gran alcance. Hay autores que apuntan que, a partir de 2005 y, especialmente, desde 2008, la comunicación local no se puede entender en Catalunya sin hacer referencia a los grupos multimedia de proximidad (GuimERÀ et al, 2009). En su opinión, uno de los elementos que ha caracterizado a este ámbito en las dos últimas décadas ha sido la progresiva consolidación de formas de concentración que han originado conglomerados privados que gestionan varios medios en un área o en diferentes zonas, los cuales han convertido los contenidos locales difundidos en varios soportes en la clave de su negocio.

Uno de los casos más evidentes de esta tendencia es el Grup Segre. Nacido sobre los cimientos de Prensa Leridana, editora del periódico que da nombre al grupo, en la actualidad cuenta con tres frecuencias de FM y cuatro concesiones de TDT. El caso del grupo ilerdense no es, sin embargo, excepcional. Diversos ejemplos repartidos por toda la geografía del país han seguido los mismo pasos, diversificando su actividad hacia la radio y la televisión: Edicions Intercomarcals, Premsa d'Osona, Publicacions Penedès, La Veu de l'Ebre...

Guimerà y sus colaboradores subrayan también la diversidad de los procesos de concentración, identificando formas poco frecuentes, como la acumulación (horizontal) de televisiones o la expansión hacia otros medios desde el audiovisual, y no desde la prensa, como ha ocurrido tradicionalmente.

A pesar del carácter multimedia de los grupos de proximidad, el eje gravitatorio de la mayoría continúa pivotando en torno a las publicaciones impresas. A pesar de la crisis que azota el sector, los medios locales parecen haber podido soportar el envite con cierta solvencia. Así, aunque las cifras de difusión muestran una tendencia al estancamiento, los números de la audiencia no dejan lugar a dudas. De forma agregada, las publicaciones integrantes de la Asociación Catalana de Prensa Comarcal, 123 títulos en 2009, cerraron el año con 875.000 lectores. Ello supone un 
incremento de 117.000 lectores respecto a las cifras del 2008. ${ }^{1} \mathrm{El} \mathrm{ranking}{ }^{2}$ lo lideran Segre y El Punt con 160.000 y 156.000 lectores, respectivamente. Les siguen cabeceras como el Diari de Tarragona con $123.000^{3}$, el 9 Nou (bisemanal) con 114.00 o Regió 7 con 58.000. Cabe subrayar también la fuerte implantación de la prensa gratuita, con cabeceras consolidadas tras haber ajustado sus tiradas iniciales a cifras más realistas (GuIMERÀ et al, 2009). Entre ellas destacan los diarios Més, con ediciones en Reus y Tarragona (92.000), y Bon dia Lleida (39.000); o los semanarios Tot Mataró, La Fura o Capgròs, que superan los 80.000 lectores.

\section{Objetivos y metodología}

En este contexto, el principal objetivo de este trabajo es identificar las estrategias de producción integrada que han desarrollado las empresas periodísticas de proximidad en Catalunya y analizar los modelos de organización de las redacciones que se han adoptado. Los resultados obtenidos forman parte de una investigación de mayor alcance que la convergencia periodística en la prensa local del territorio.

La complejidad que se deriva de los procesos de convergencia mediática exige aproximaciones al fenómeno también plurales. Es por este motivo por lo que, una vez definidos los objetivos, se optó por una combinación de métodos cuantitativos y cualitativos; en concreto, la realización de una encuesta y de entrevistas en profundidad. En esta decisión influyó la convicción de que el uso de parámetros exclusivamente cuantitativos ofrecía limitaciones para explicar en profundidad la realidad del fenómeno estudiado (SÁDABA et al, 2008).

Para el enfoque cuantitativo, se realizó una encuesta a los responsables de los 123 medios adscritos a l'Assocació Catalana de Premsa Comarcal, organización que acoge la mayoría de los medios de proximidad de pago editados en Cataluña. Con esa finalidad se elaboró un cuestionario compuesto por 19 preguntas, la mayoría cerradas, agrupadas es cinco grandes bloques: "Producción periodística y convergencia”, "Periodista polivalente", "Distribución multiplataforma”, "Audiencias Activas" y "Otros", que incluía preguntas sobre uso de fuentes y aspectos éticos. Los resultados obtenidos en el primer bloque serán fundamentalmente los usados para el presente trabajo.

El formulario, que estaba disponible en internet, se distribuyó entre todos los directores de las publicaciones, que dispusieron de dos meses para responder (noviembre-diciembre 2008). Para asegurar un índice mayor de retorno, a mediados del período de recogida de respuestas, se contactó telefónicamente con los directores para animarlos a completar la encuesta. Finalmente, se recogieron 56 respuestas válidas, lo que supone un porcentaje de respuestas del $45 \%$.

Para completar los resultados cuantitativos, se mantuvieran entrevistas en profundidad con miembros de las plantillas de diversos medios de comunicación seleccionados durante el primer semestre de 2009. No existe unanimidad con el

${ }^{1}$ Datos proporcionados por el Baròmetre de la Comunicació i la Cultura.

2 Sexta oleada de 2009 del Baròmetre de la Comunicació i la Cultura

${ }^{3}$ Diari de Tarragona no forma parte de la ACPC 
número de entrevistas recomendable para abordar estudios de esta naturaleza. La variabilidad es amplia, desplazándose entre las 130 entrevistas que mantuvo Jane SINGER (2004) en su investigación sobre la convergencia en los Estados Unidos, a poco más de la decena que ofrecen un amplio número de trabajos (GARCía Aviles; 2007, DupAGNe y GARRISON; 2006, QuinN; 2006).

Para esta investigación se realizaron 19 entrevistas en profundidad a miembros de ocho grupos de comunicación. Como en estudios anteriores (BoczKowsKI, 2004; BOCZKOWSKI y FERRIS, 2005; KLINENBERG, 2005; ERDAL, 2009), se centró el análisis en corporaciones empresariales y no en alguno de sus medios aisladamente, porque la convergencia está muy vinculada a los conglomerados multimedia.

Para la selección de los medios se tuvieron en cuenta diversos criterios con el fin de obtener la máxima y mejor representatividad del fenómeno. Así, se consideró la diversidad geográfica -se seleccionaron grupos de las cuatro demarcaciones catalanas-; la diversidad mediática -grupos con estructuras diferentes y amplia representación de medios con distintos niveles de desarrollo-; la diversidad de periodicidad -desde medios diarios hasta revistas mensuales-; y la diversidad en la tirada de los medios impresos de cada grupo ${ }^{4}$-desde 30.000 ejemplares a 1.800 .

Tras la toma en consideración de las variantes mencionadas, se decidió entrevistar a trabajadores de los grupos siguientes: Grup Segre, El Punt, El 9 Nou, Regió 7, La Veu de l'Ebre, Revista Cambrils, Capgròs y OAMCIC (Cuadro 1). Excepto en el caso de los dos últimos grupos, las publicaciones impresas del resto pertenecen a la ACPC. Se añadió Capgròs y OAMCIC porque ofrecían experiencias interesantes entorno a la convergencia en virtud de su originalidad, novedad o incidencia en la audiencia. Un grupo de prensa gratuita con fuerte implantación en internet (Capgròs) y una corporación pública de carácter audiovisual.

Cuadro 1. Grupos analizados

\begin{tabular}{|l|c|r|l|}
\hline \multicolumn{1}{|c|}{ Grupo } & Periodicidad & \multicolumn{1}{c|}{ Tirada $^{\mathbf{1}}$} & \multicolumn{1}{c|}{ Medios involucrados } \\
\hline Grup Segre & Diaria & 25.084 & Prensa, web, televisión, radio, e-paper \\
\hline El Punt - Girona & Diaria & 21.500 & Prensa, web \\
\hline El 9 Nou - Vic & Bisemanal & 11.500 & Prensa, web, televisión \\
\hline Regió 7 & Diaria & 9.908 & Prensa, web, televisión \\
\hline La Veu de l'Ebre & Semanal & 6.500 & Prensa, web, televisión, radio \\
\hline Revista Cambrils & Mensual & 1.800 & Prensa, web \\
\hline Capgròs & Semanal & 30.000 & Prensa, web \\
\hline OAMCIC & - & - & Web, radio, televisión \\
\hline
\end{tabular}

Para disponer de la máxima variedad de puntos de vista, en la selección de las personas a entrevistar se intentó que estuviesen representados los máximos responsables de cada uno de los grupos, así como los periodistas involucrados en procesos de convergencia. Finalmente, se mantuvieron extensas conversaciones con

\footnotetext{
${ }^{4}$ A pesar de tomar como unidad de análisis los grupos, no puede perderse de vista que en la mayoría de casos el origen de dichas compañías se sitúa una publicación impresa.

${ }^{5}$ Según información de la web de l’ACPC: http://www.premsacomarcal.cat
} 
19 personas. El modelo de entrevista utilizado fue el semiestructurado, ya que posibilita un cierto control sobre las respuestas del interlocutor, pero al mismo tiempo permite un elevado grado de libertad al entrevistador y al entrevistado para mantener una conversación distendida. Las entrevistas partían de un cuestionario previo que ejercía de hilo conductor que garantizaba la obtención de la información requerida, sin que ello impidiese que los informantes derivasen el diálogo hacia otros aspectos igualmente relevantes en torno al objeto de la investigación. Todas las conversaciones fueron grabadas.

\section{Resultados}

\subsection{Fábricas de información}

Una de las primeras conclusiones que se obtienen en cualquier estudio sobre la prensa local y comarcal es que estamos ante un sector muy heterogéneo, con muchas particularidades, realidades distintas y, en ocasiones, delicadas; donde generalizar no es fácil, ni recomendable. También conviene recordar la complejidad del fenómeno, que puede adoptar diversas formas y que incide en los grupos de comunicación a muy diversas esferas. En este sentido, Juan CAL, máximo responsable del grupo Segre, habla de, al menos, dos tipos de convergencia entre medios locales, la periodística y la empresarial.

Aunque este trabajo se centra particularmente en la periodística y su incidencia en la organización de las redacciones, lo cierto es que no puede desvincularse una de la otra. Los directivos entrevistados subrayan los beneficios económicos que de ellas se derivan. Todos admiten que este fenómeno comporta una nueva manera de pensar y trabajar en sus empresas. No obstante, reconocen que en conjunto esos cambios se han asimilado bien porque permiten ahorrar dinero para ofrecer los mismos (o más) productos que antes. Además, posibilita una mejora en la eficiencia de la gestión, un aumento de los anunciantes -aunque en este punto no todos coinciden- y un incremento de visibilidad en el mercado.

Entre los entrevistados existe un cierto consenso en considerar sus respectivos medios como pequeñas "fábricas de información o de contenidos"; y a ellos mismo como operarios de esas fábricas. El concepto no es nuevo. Telecinco fue uno de los primeros medios de comunicación en España en reivindicar esa terminología, al usarlo para referirse a sus servicios informativos. A pesar de no estar exento de connotaciones negativas, la realidad parece imponer una concepción global de la producción informativa, en la cual los periodistas de un medio puedan dar salida a sus informaciones a través de diversos canales. El énfasis se traslada de la plataforma a los contenidos.

El responsable del canal de televisión de Regió $7^{6}$ introduce un elemento fundamental para entender esta nueva concepción: "Hay que pensar en términos de grupo de comunicación”. Efectivamente, para poner en funcionamiento alguna forma de coordinación entre redacciones es preciso que los periodistas se sientan como parte

\footnotetext{
${ }^{6}$ Regió 7 tiene una difusión de unos 8.500 ejemplares diarios, con fuerte implantación en las comarcas de la Catalunya central. Actualmente dispone también de un cibermedio y un canal de televisión.
} 
de un grupo de comunicación, y no exclusivamente como trabajadores de un único medio. Los entrevistados coinciden en afirmar que no es sencillo, existen problemas y reticencias y es difícil romper con las dinámicas largamente instaladas.

La encuesta realizada a los miembros de la ACPC revela que sólo el 14,28\% de los medios que la respondieron elaboran sus contenidos en colaboración con terceros. Los resultados destacan por su variedad, ya que entre los que respondieron de forma positiva se encuentran publicaciones diarias -El Punt, Segre-, semanales La Veu de l'Ebre o Nova Tàrrega-, o de frecuencia mensual -El Ter o El Marginet. Del mismo modo, la variabilidad es muy amplia en cuanto a la difusión que representan, que oscila entre los 26.000 de El Punt a los 600 ejemplares de El Martinet. Sería incorrecto, por tanto, restringir esta estrategia sólo a los medios que presentan un conjunto de características específicas.

Aunque el porcentaje podría considerarse modesto, no puede perderse de vista las particularidades de la prensa de proximidad catalana y su relación con internet. Las publicaciones más modestas -la mayoría- no disponen de versión digital y si existen son meros escaparates de la publicación impresa, ofreciendo versiones en PDF de ejemplares anteriores y sin actualización continuada de contenidos. El presidente de la Associació Catalana de Premsa Comarcal (ACPC), Estanis Alcover, lo resume con la frase: "hasta ahora, parece que estuviesen peleados"; para acabar añadiendo que entre los editores de algunas publicaciones especialmente las más humildes- ofrecer de forma gratuita por la red los contenidos que otros pagan por tenerlos en papel es una falta de respeto al lector.

El propio Alcover ofrece una posible explicación a esta actitud: la endeble estructura empresarial de la mayor parte de las publicaciones locales, que sobreviven en un inestable equilibro entre el amateurismo y la profesionalización. Algunas empresas gestionan una única cabecera, la publicación impresa. Cabe subrayar, sin embargo, que en ocasiones, el rechazo también proviene de medios consolidados, que gozan de una importante implantación en su ámbito de influencia. Es el caso del Diari de Vilanova, ${ }^{7}$ que evita tener una gran presencia en la red argumentando que el papel ya funciona suficientemente bien; o de El Punt, ${ }^{8}$ uno de los principales rotativos catalanes y que hasta mediados de 2009 desarrolló una modestísima actividad en internet.

En los medios de mayor envergadura las reticencias hacia internet -aunque han existido- tienden a desaparecer y ya han incorporado las ediciones digitales en sus estrategias multimedia. En este sentido, el director general de El 9 Nou, Jordi Molet, reconoce que inicialmente le preocupaba que internet fagocitase la versión impresa, por lo que su página en internet se limitaba a ofrecer la portada del día. Actualmente, esta fobia se ha esfumando y han comprobado que ni la red, ni la televisión perjudican el periódico.

Excepto en un caso, Nova Tàrrega, la colaboración entre medios siempre está

\footnotetext{
${ }^{7}$ Publicación semanal con una difusión ligeramente superior a los 6.000 ejemplares.

${ }^{8}$ Diario con una difusión superior a los 26.000 ejemplares diarios. Publica ediciones en Barcelona, Girona y Tarragona. http:www.elpunt.cat.
} 
protagonizada por las versiones en papel y las digitales, como lo demuestran las sinergias generadas entre Segre.com, Ebredigital.cat o El9nou.cat con Segre, La Veu de l'Ebre y El 9 Nou, respectivamente. Con todo, también hay ejemplos de colaboración más complejos, en los que intervienen además medios audiovisuales del propio grupo. Son las experiencias desarrolladas por El Ter y Ràdio Manlleu, o los ya mencionados La Veu de l'Ebre y Segre, con sus respectivas emisoras, Imagina Ràdio y Ràdio Segre. Además, tanto el periódico leridano como El 9 Nou recorren habitualmente a la confluencia con sus televisiones: Segre TV y El 9 TV.

En algunos casos, las publicaciones aprovechan las sinergias con otras cabeceras en papel del mismo grupo empresarial para elaborar contenidos en colaboración. Nova Tàrrega y Segre o Regió 7 y el Diari de Girona optan por esta vía. Contrariamente a lo que se podría pensar, no se trata de una fórmula reciente, sino que se realiza de manera más o menos sistemática desde hace más de una década: de 1989 entre Nova Tàrrega y Segre, o de 1983 entre El Ter y Ràdio Manlleu.

A pesar de la variedad de las fórmulas adoptadas, la coordinación entre plataformas se suele producir en condiciones de superioridad del medio "original", respecto al resto; y muy particularmente respecto al medio digital. Con todo, precisamente la irrupción de internet y la voluntad de potenciación de las ediciones digitales ha sido el detonante real que ha desencadenado las primeras iniciativas de convergencia. Es el caso de la Revista de Cambrils, que en el momento que ingresaba en el mundo virtual realizaba los primeros pasos hacia la convergencia y la profesionalización. También el anuncio de la puesta en marcha del cibermedio fue el resorte que puso en marcha la convergencia en El Punt.

La diversidad de modelos de funcionamiento entre plataformas es amplia. En ciertos casos, el motor de la convergencia no ha sido internet, sino la televisión. Es el caso de El $9 \mathrm{Nou}$, que inició el proceso de convergencia entre medios con la puesta en funcionamiento en 2004 de la televisión, o de La Veu de l'Ebre donde, según su máximo responsable, la concesión de una licencia de TDT forzará las sinergias entre plataformas.

Sin embargo, el caso más paradigmático es el del Grupo Segre, que a pesar de tener su origen en el periódico impreso que da nombre al grupo, desde los años noventa trabaja con una clara orientación hacia la multimedialidad. En primer lugar, con una emisora de radio (actualmente con tres frecuencias y dos programaciones) y a partir de 2001, con una televisión (inicialmente analógica y ahora con cuatro concesiones de TDT). Pero ha sido internet el verdadero desencadenante e impulsor de la convergencia periodística. En palabras del máximo responsable, Juan CAL, la web está situada "en el centro", ya que es aquí donde se puede trabajar realmente hacia la convergencia de contenidos.

Sólo el 23,2\% de los medios encuestados reconocen la existencia de alguna forma de distribución multiplataforma. En la mayoría de los casos son medios que ya colaboran a nivel de producción y que buscan diversificar los canales de difusión. Como suele suceder en estos casos, los contenidos se distribuyen entre medios del propio grupo empresarial. Se observa en el $84,6 \%$ de los ejemplos.

De las cuatro posibles formas de distribución que se definieron en la encuesta - 
promoción de medios, promoción de contenidos, reproducción exacta de contenidos y adaptación de contenidos- es esta última la fórmula más popular. Está presente en el $72.7 \%$ de los casos (Gráfico 1).

Gráfico 1. Fórmulas de distribución de contenidos multiplataforma (Elaboración propia)

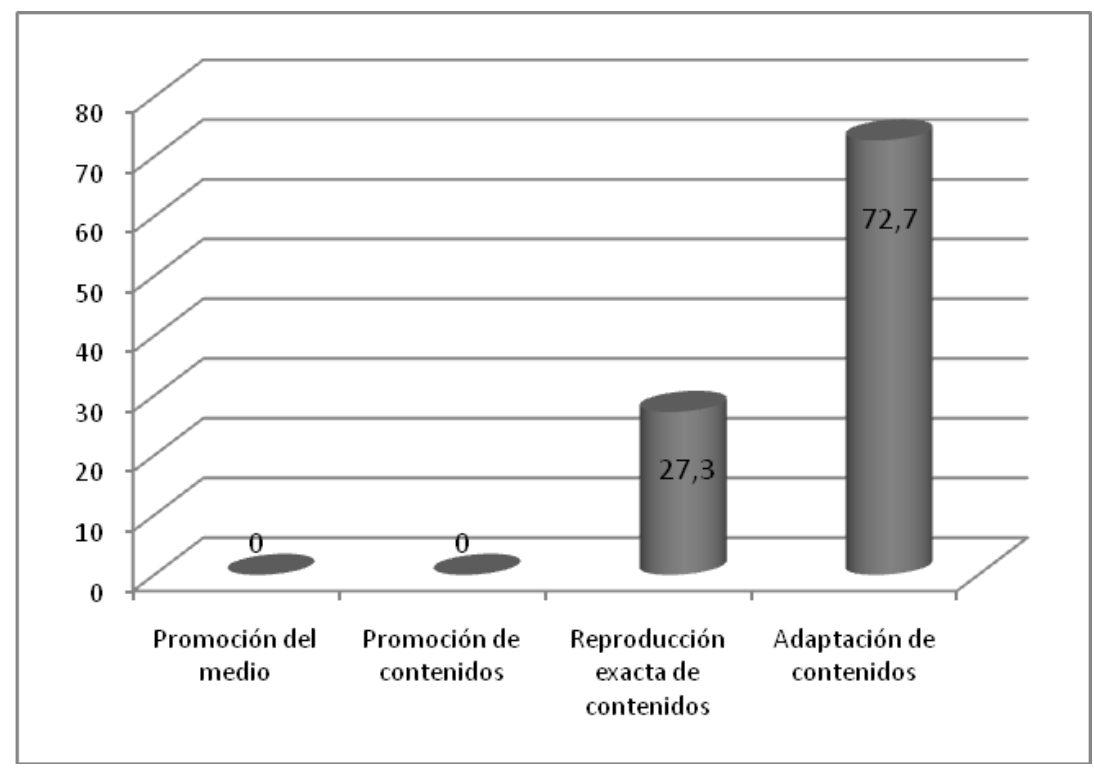

\subsection{Producción periodística y organización de las redacciones}

En términos generales, cuando los entrevistados se refieren a la convergencia periodística piensan en redacciones integradas o, al menos, en redacciones coordinadas. A pesar de ello, la convergencia periodística no siempre pasa por la integración de redacciones, aunque sí que se materializa en alguna forma de producción integrada.

Los resultados obtenidos en la encuesta muestran que la manera de organización de las redacciones se reparte de forma equilibrada entre las unificadas y las que se mantienen separadas, aunque con una intensa coordinación entre ellas. Entre las primeras nos encontramos los casos de, por ejemplo, El 9 nou con el el9nou.cat; y Segre con Segre.cat y Lleida.com. En ocasiones a las ediciones impresa y digital se les añade la emisora de radio; como El Ter, ElTer.net y Ràdio Manlleu; o La Veu de l'Ebre con Ebredigital y Imagina Ràdio.

Como se observa en el gráfico 2, tan popular como las redacciones únicas son las redacciones que se mantienen separadas, entre ellas existe una estrecha relación y la coordinación es tarea cotidiana. Cuando la manera de operar es esta, suele intervenir la televisión. Así, El 9 Nou y de El 9 Nou TV disponen de redacciones separadas, como ocurre también en Segre y Segre TV. En el resto de los casos, las redacciones funcionan de forma independiente, lo que no impide que se puedan compartir contenidos, aprovechando las dinámicas propias del grupo. 
Gráfico 2. Estructura de las redacciones (Elaboración propia)

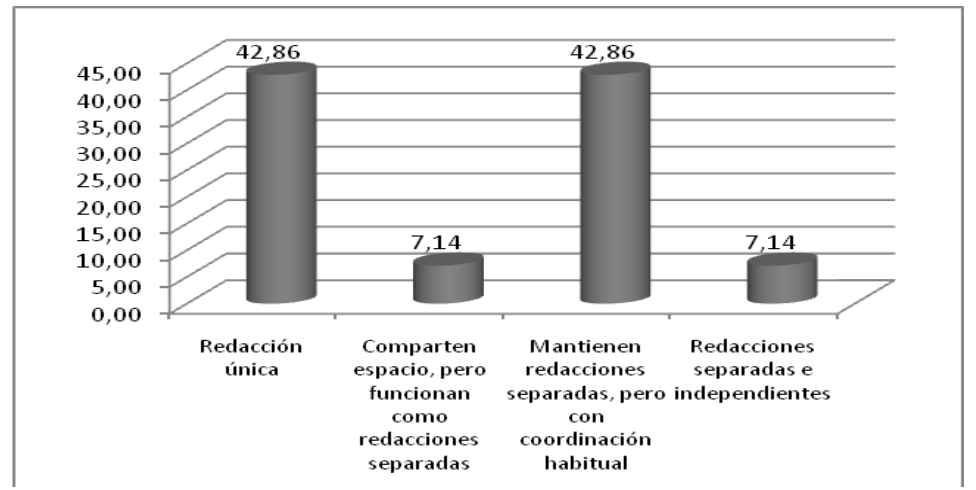

Desde el punto de vista operativo, la confluencia de redacciones puede ser la solución más sencilla en un medio de dimensiones modestas. Y algunos de los medios analizados son buenos ejemplos de ello.

\subsubsection{Redacciones unificadas}

En los medios que cuentan con una redacción única, los periodistas suelen elaborar contenidos indistintamente para cualquier plataforma, sin que haya, en principio, un claro predominio de una sobre las otras. En la práctica, sin embargo, la ascendencia de la cabecera matriz en los medios de proximidad es todavía importante y los mayores esfuerzos se suelen volcar sobre la edición impresa. Quizá la publicación que mejor ha conseguido romper con esa dinámica ha sido la Revista de Cambrils. A pesar de la preeminencia del papel, su voluntad de potenciar su presencia en internet, así como la periodicidad mensual del producto impreso, han facilitado que la prioridad del día a día se haya centrado en la web, que se actualiza diariamente. Esta nueva concepción se ilustra con el subtítulo a la cabecera tradicional que se ha añadido en la edición en internet: Diari digital.

En opinión del director de la revista y de dos de sus redactoras, el impulso de la versión digital ha incidido positivamente en la impresa. La obligación de alimentar diariamente el cibermedio -se marcaron una cifra mínima de cuatro informaciones nuevas al día- revierte en la calidad en la medida que les obliga a buscar noticias en lugar de esperar a las convocatoria de prensa o notas oficiales. Además, las informaciones publicadas originalmente en internet son recuperadas y reelaboradas según la importancia que han tenido en perspectiva, para ser finalmente incluidas en la revista en papel.

Así, pues, para la publicación tarraconense, ambas plataformas se complementan a la perfección, permitiendo llegar a más audiencia; aunque no dejan de reconocer que es el suporte tradicional la que genera la principal fuente de ingresos de la empresa.

Otro ejemplo relevante de redacciones integradas lo encontramos en un grupo de comunicación multimedia, y que por tanto no forma parte de la ACPC. Se trata de Cugat.cat, el portal informativo del Ayuntamiento de Sant Cugat (Barcelona). Probablemente, este sea el único caso que realmente funcione como una redacción integrada, multiplataforma y sin predominio de ningún medio. Según indica su 
directora, Mónica Lablanca, en función de la noticia y el momento del día en que se produce se decide la plataforma de difusión.

Con la misma filosofía se puso en marcha la edición digital de El Punt, inaugurada en febrero de 2009 sin contratar a más periodistas. Los redactores de la versión impresa producen también para el cibermedio. Cada responsable de área decide quién cubre un determinado acontecimiento, para qué soporte y en qué formato: un flash informativo que se cuelga rápidamente en la web, una pieza más desarrollada para la web o si se reserva para el papel y se incorpora el cibermedio con posterioridad.

Este modo de operar incide de forma directa en el funcionamiento de las redacciones. Obliga a reorganizar los turnos y las tareas asignadas. Los responsables de cierre posponen su horario de entrada y asumen competencias propias de un cibermedio, velando por la actualización de la edición digital en los momentos en que la edición en papel ya está cerrada.

La necesidad de producir para dos o más plataformas incide también en la carga de trabajo de los periodistas. En opinión del redactor d'El Punt Eduard Batlle, de momento, es asumible que un periodista elabore información para web y papel. Aunque matiza que lo es porque existe una estructura superior formada por los jefes de sección que lo facilita. De todas formas, profesionalmente les exige adoptar una actitud mucho más activa y estar pendientes de lo que sucede en todo momento.

El director de El Punt, Emili GISPERT, afirma que la convergencia entre redacciones ha sido una prioridad desde el primer día, aunque reconoce que en la actual situación de crisis ha aumentado "la fe" en este proceso, "haciendo de la necesidad, virtud”. GISPERT sentencia que el futuro pasa por la convergencia y por internet, aunque no duda en afirmar que las empresas periodísticas todavía habrán de vivir unos cuantos años del papel.

Como en El Punt, la producción integrada en Cugat.cat supone una reorganización de las tareas. La redactora Mar CASTRO explica que ahora es esencial una mayor racionalidad en el número de noticias que se cubren a lo largo de la jornada. Admite que se tarda más en realizar información multimedia, por lo que el número de piezas asignadas deberá ser menor si no se quiere incidir en la calidad. "Si antes hacía cinco piezas, ahora en el mismo tiempo hago tres, siempre y cuando no todas tengan vídeo", ejemplifica.

La voluntad de potenciar la inmediatez que ofrece internet comporta en algunos medios otros cambios que van más allá de la mera reorganización de las redacciones y que, hasta cierto punto, podrían considerarse contradictorios: el teletrabajo. En El Punt caminan en esa dirección y han dotado a algunos de sus reporteros de equipos ligeros de producción que hacen posible que manden las informaciones directamente desde el lugar de la noticia. Además de ganar en inmediatez, lo hacen en agilidad -defiende el director- permitiendo que los periodistas puedan cubrir diversos actos sin tener que pasar por la redacción.

Esta fórmula forma parte de una estrategia más amplia que permite un ahorro de costos importante. La fuerte implantación territorial de El Punt y su funcionamiento basado en las ediciones locales había obligado a disponer de hasta 22 corresponsalías 
con sus respectivas sedes. Lo que a parecer de los directivos del grupo suponían unos costes inasumibles. El teletrabajo ha permitido el cierre de algunas de esas sedes. El director del rotativo, Emili GISPERT, cree que la tendencia será disponer de una sede central, y otras satélites únicamente allí donde sea estrictamente necesario, donde se realizarán tareas de edición, cierre, dirección... A la larga, los puestos de trabajo deberán ser distintos, "no será necesario tener lugar para cada uno, sino espacio para que la gente pueda instalarse, conectarse y trabajar”.

Desde el punto de vista laboral, se pactó con el comité de empresa que los trabajadores que se acogiesen a esta experiencia lo hiciesen de forma voluntaria. En los primeros cuatro meses de funcionamiento optaron por el teletrabajo una treintena de trabajadores. A todos ellos se les facilitó ordenador, conexión a internet, teléfono móvil, cámara fotográfica, escáner... y la posibilidad de trabajar desde el lugar de la noticia y en sus domicilios.

\subsubsection{Redacciones coordinadas}

Si en las redacciones integradas los periodistas elaboran indistintamente contenidos para cualquier plataforma, en las redacciones compartidas los trabajadores comparten espacios, pero mantienen un medio prioritario para el que elaborar las informaciones. En ellas, sin embargo, existe algún tipo de coordinación que puede ser más o menos intensa e institucionalizada según cada medio.

Uno de los mejores ejemplos de este tipo de redacciones la encontramos en el grupo Segre. En el conglomerado leridano la puesta en funcionamiento de Segre.com fue la excusa para que confluyesen tres plataformas ya existentes: el periódico, la emisora de radio y la televisión. Todas ellas comparten espacio, pero mantienen su independencia. Se consideran redacciones diferenciadas porque tienen ritmos distintos, no porque funcionen de forma distinta.

La coordinación entre las diversas redacciones está garantizada gracias a la existencia de un equipo formado por un subdirector del periódico, los directores de los servicios informativos de la radio y de la televisión, el coordinador de internet y el jefe de fotografía; a modo del superdesk que han desarrollado diversos medios (Clarín, The Daily Telegraph...). Este equipo se reúne regularmente para establecer las asignaciones y evitar duplicidades. A pesar de que los redactores trabajan primordialmente para un único medio, pueden también elaborar informaciones para cualquier otra plataforma. Además, hay periodistas del diario que colaboran en programas de televisión en calidad de expertos.

La colaboración entre redacciones se plasma de la forma más evidente en el portal informativo del grupo leridano. A pesar de ser el detonante de la estrategia de convergencia del grupo, su cibermedio no dispone de redactores propios, únicamente tiene dos trabajadores: un informático y un editor-responsable. Los contenidos son producidos por los periodistas de los otros medios del grupo.

El director del grupo, Juan CAL, defiende la organización actual basada en la coordinación entre redacciones, aunque asegura que en el futuro se deberían plantear las estructuras de las redacciones partiendo de parámetros distintos y dividir las redacciones en dos ámbitos: calle o mesa, o urgencia versus profundidad. 
La estructura de Segre es compartida por otros medios. En el 9 Nou existen equipos base para cada medio, aunque todos los periodistas pueden producir para el resto de plataformas. Los contenidos del web provienen de la labor realizada por los periodistas de prensa y de televisión, y un redactor se encarga de editar las piezas para el cibermedio e incorporar noticias de última hora.

La relación entre ambas redacciones es técnicamente sencilla en la medida que existe una red integrada. En general, existe una diferenciación clara de las tareas entre los periodistas del periódico y los de televisión. El contacto con muchas fuentes o una investigación muy elaborada difícilmente recae en la televisión, sino que se realiza desde el periódico, con mayor número de efectivos. La redacción de televisión se ahorra estas tareas previas, aunque obviamente asume la elaboración de las piezas utilizando sus propias imágenes y la información proporcionadas por los compañeros del papel.

Las sinergias y la colaboración entre las plataformas del grupo van más allá de la elaboración de información diaria. Así, el jefe de la sección de política y economía del periódico dirige y presenta un debate semanal de temática política en El 9 Nou TV; y el jefe de deportes un informativo que se emite los lunes.

La diversidad de modelos que permite la convergencia se observa también en Regió 7 o La Veu de l'Ebre, donde se comparten espacios, pero no se aprecian niveles de coordinación tan intensos como en los casos precedentes. Ello no supone que sus modelos sean menos válidos, sencillamente desarrollan una solución que puede ser la óptima para las características y los objetivos de los grupos que las aplican.

En Regió 7, la televisión, el periódico y el web comparten espacios, pero las redacciones funcionan de forma totalmente independiente y las colaboraciones entre ellas se suelen reducir a colaboraciones puntuales. El gerente del grupo, Fèlix Noguera, defiende la producción separada porque opina que las diferencias entre soportes son lo suficientemente importantes como para trabajar de esta manera. El director de la televisión, sin embargo, cree que sería interesante intensificar las relaciones entre redacciones, por ejemplo estableciendo mecanismos formales de intercambio de información. Técnicamente sería sencillo puesto que las dos redacciones ya comparten sistemas redaccionales y redes de trabajo.

\subsubsection{Redacciones integradas a la fuerza}

Un caso distinto es el de La Veu de l'Ebre, un semanario con fuerte implantación en las comarcas del sur de Tarragona y que, a pesar del carácter multimedia del grupo, el protagonismo del semanario es absoluto. La redacción del papel y de internet es única, mientras que la emisora de radio dispone de su propio espacio. De todas formas, los boletines informativos de la emisora se elaboran en la redacción del semanario y se locutan desde esa misma redacción, donde se ha instalado un pequeño estudio de radio para facilitar este nivel de polivalencia mediática. Esta estructura redaccional parece estar más marcada por las necesidades que por la voluntad de sus máximos responsables, que preferirían una redacción propia para la radio si las condiciones financieras lo permitiesen. La misma solución se intuye para la futura emisora de televisión, prevista tras la concesión de una licencia de TDT. 


\section{Actitudes y estrategias ante la convergencia}

Reorganizaciones como las descritas suelen ser motivo de problemas o, cuando menos, generan reticencias. El proceso puede ser traumático para los implicados. Es por ello que la mayoría de los entrevistados coinciden en la necesidad de obtener la complicidad de los trabajadores antes de poner en marcha iniciativas de convergencia de redacciones.

Para reducir las resistencias, las empresas fundamentalmente pueden adoptar dos posiciones: el liderazgo o la persuasión. La primera se basa en la jerarquía y el uso de la estructura de poder; dicho de otro modo: la imposición. La segunda, en cambio, persigue que los periodistas perciban las ventajas que ocasionará en su quehacer diario la adopción de las innovaciones propuestas, para que, a través de la participación, las hagan suyas.

La persuasión es la opción escogida por la mayor parte de los medios analizados. Así lo verbalizan responsables y trabajadores de El Punt o La Veu de l'Ebre, por poner un par de ejemplos. En Cugat.cat la integración se realizó de forma vertical. Las decisiones estratégicas se tomaron desde la dirección, aunque los dirigentes del ente público que lo gestionan matizan que siempre intentaron buscar el consenso. Para ello se creó un grupo de trabajo que involucrase a los trabajadores en todo el proceso.

A pesar de las estrategias adoptadas, reformas en las maneras de trabajar generan incertidumbre y temor. Temor en convertirse en "hombres orquesta", a la sobrecarga de trabajo, a un descenso de la calidad del producto, a una falta de reconocimiento profesional y salarial... Miedos, todos ellos, perfectamente identificados en otros estudios de características similares (MAsIP y Micó, 2009).

Para reducir el malestar entre los trabajadores, es obligación de la empresa proporcionar herramientas efectivas "que generan automatismos", así como "formación y complicidad”, apunta la directora de Cugat.cat. Para Mónica LABLANCA, los periodistas veteranos suelen ser los que ofrecen más resistencias, obligados a asumir nuevos retos para los cuales no estaban ni concienciados, ni preparados. El máximo responsable del Grupo Segre comparte el diagnóstico y subraya la dificultad de extirpar "castas, jerarquías enquistadas que dificultan ciertos cambios."

Con pocas excepciones, los medios locales y comarcales no han organizado grandes planes de formación para que sus trabajadores afronten en condiciones las exigencias propias del nuevo entorno. Muchos de los entrevistados alegan que sus empleados ya tienen unas nociones básicas gracias a las Facultades de comunicación. Otro grupo de directivos se conforma con indicaciones prácticas sobre el manejo de nuevos equipos tecnológicos y software concretos. Mientras que sólo un tercer grupo apela por la utilidad y la necesidad de la formación continuada.

\section{Conclusiones}

La diversidad que caracteriza la prensa local y comarcal catalana se pone también de manifiesto cuando se analiza las iniciativas de convergencia que desarrollan. Para la mayoría, pequeñas publicaciones hiperlocales con un fuerte componente de amateurismo, la convergencia suena a entelequia. Junto a ellas, un número reducido de cabeceras, pero que aglutinan la mayor parte de la audiencia del sector, han 
empezado a experimentar con nuevas formas de organización de las redacciones y de distribución de contenidos.

Entre los medios locales estudiados, se encuentran redacciones integradas que trabajan de forma unitaria y producen para más de una plataforma, habitualmente el papel y la web. Cuando participan medios audiovisuales -radio y/o televisión- la fusión real es excepcional, aunque en ocasiones pueden compartir espacios. A pesar de las dificultades, se intentan establecer mecanismo de coordinación entre redacciones, como las llamadas mesas multimedia. Desde ellas se establecen las asignaciones y se decide la cobertura multiplataforma de los hechos noticiosos. En la mayor parte de los casos, los periodistas tienden a elaborar informaciones para un único medio, que tiene carácter prioritario.

En la mayoría de los medios como los analizados, la implantación de fórmulas de producción integrada, o de otras medidas como la generalización de la figura del periodista polivalente, suele obedecer, por sus dimensiones, a una necesidad, que, como dicta el tópico, se reconvierte en virtud. Más allá de las ventajas -que las hayy de las críticas -que también existen-, los medios locales catalanes que han diversificado sus actividades perciben la convergencia como una tabla de salvación que les permite mantener una gestión eficiente en un entorno frágil. Aunque algunos no niegan que una planificación inadecuada y el peligro de sucumbir ante la generalización de los "periodistas orquesta" puede incidir negativamente en la calidad de los productos, otros opinan que sería el peaje a pagar por mantener unos medios de proximidad arraigados al territorio y económicamente viables, especialmente si son audiovisuales.

En tercer lugar, es necesario resaltar que la problemática relación existente en el seno de los medios locales entre internet y el papel, aunque en la actualidad los recelos existentes tienden a diluirse, particularmente entre los de mayores dimensiones. Entre estos, obviar las posibilidades que ofrece el ciberespacio equivale a permitir que la parcela vacante la ocupe la competencia. Un buen ejemplo de ello sería uno de los medios estudiados, Capgròs, una publicación que ha adquirido una fuerte implantación en su comarca e expensas de la actitud dubitativa de El Punt en internet.

Desde un punto de vista estrictamente periodístico, la coexistencia de los dos soportes -el analógico y el digital- tiene una incidencia directa y mutua en los contendidos que se producen para cada plataforma. Una incidencia, sin embargo, de características distintas según el carácter de las publicaciones. Entre las diarias, las versiones electrónicas son claramente subsidiarias -a pesar de algunos discursos-. Los contenidos publicados en ellas son reelaboraciones o versiones previas de las que aparecerán en la edición en papel del día siguiente. En la mayoría, la redacción es de mínimos, con uno o dos redactores que se nutren de lo aportado por los colegas del papel.

Entre las publicaciones de periodicidad menor -semanal o mensual-, la necesidad de mantener actualizado el cibermedio antes de la aparición de cada nuevo ejemplar impreso acaba repercutiendo en el periódico en papel. Revista de Cambrils y Capgròs son buenos ejemplos de lo descrito. 


\section{Referencias bibliográficas}

BOCZKOWSKI, Pablo J. (2004): Digitizing in Online Newspapers. Cambridge, The MIT press.

BOCZKOWSKI, Pablo J.; FERRIS, José A. (2005). "Multiple Media, Convergent Processes, and Divergent Products: Organizational Innovation in Digital Media Production at a European Firm. The Annals of the American Academy of Political and Social Science, vol. 597, pp. 32-47.

DAILEY, Larry;, DEMO, Lori; SPILLAN, Mary (2005): "The Convergence Continuum: A Model for Studying Collaboration Between Media Newsrooms”. Atlantic Journal of Communication, vol. 13, pp. 150-168.

DUPAGNE, Michel y GARRISON, Bruce (2006): "The meaning and influence of convergence. A qualitative case study of newsroom work at the Tampa News Center” Journalism Studies, vol. 7, pp. 237-255.

ERDAL, Ivar John (2009): “Cross-Media (Re)Production Cultures”. Convergence: TheInternational Journal of Research into New Media Technologies, vol. 15, pp. 215-231.

GARCÍA AVILÉS, Juan Alberto (2007): “Estándares profesionales en la convergencia de redacciones multimedia. Hacia una cultura periodística convergente". En: MASIP, Pere.; ROM, J. (eds.): Las encrucijadas de la comunicación: límites y transgresiones. Actas del IV Congreso Internacional Comunicación y Realidad. Barcelona, Trípodos, pp. 345-357

GARCÍA AVILÉS, Juan Alberto et al (2009): “Integración de redacciones en Austria, España y Alemania: modelos de convergencia de medios” Anàlisi. N. 38, p. 173-198.

GUIMERÀ, Josep Àngel et al (2008): La comunicació local a Catalunya: informe 2008. Bellaterra, UAB. http://www.portalcomunicacion.com/ocl/down/ infome_comloc_2008.pdf [Consulta: 22/05/09]

KLINENBERG, Eric (2005): "Convergence: News Production in a Digital Age”. The Annals of the American Academy of Political and Social Science, vol. 597, pp. 48-64.

MASIP, Pere y MICÓ, Josep Lluís (2009): “El periodista polivalent en el marc de la convergència empresarial”. Quaderns del CAC, núm. 31-32, p. 85-92

QUINN, Stephen (2006): Conversations on convergence. New York, Peter Lang.

SÁDABA, Charo et al (2008): "Métodos de investigación sobre convergencia periodística”. En: DIAZ NOCI, Javier y PALACIOS, Manuel (ed.) Metodologia para o estudio dos cibermeios. Estado da arte \& perspetivas. Salvador: Universidade Federal da Bahia, 2008.

SALAVERRÍA, Ramón y NEGREDO, Samuel (2008): Periodismo integrado: convergencia de medios y reorganización de redacciones. Barcelona, Sol 90.

SINGER, Jane B. (2004): "Strange Bedfellows? The Diffusion of Convergence in Four News Organizations”. Journalism Studies, vol. 5, núm. 1, pp. 3-18. 